Proyecciones Journal of Mathematics Vol. 38, No 1, pp. 1-11, March 2019.

Universidad Católica del Norte

Antofagasta - Chile

\title{
Odd harmonious labeling of super subdivision graphs
}

\author{
P. Jeyanthi \\ Govindammal Aditanar College for Women, India \\ S. Philo \\ Manonmaniam Sundaranar University, India \\ and \\ M. K. Siddiqui \\ COMSATS University Islamabad, Pakistan \\ Received : December 2015. Accepted : November 2018
}

\begin{abstract}
A graph $G(p, q)$ is said to be odd harmonious if there exists an injection $f: V(G) \rightarrow\{0,1,2, \cdots, 2 q-1\}$ such that the induced function $f^{*}: E(G) \rightarrow\{1,3, \cdots, 2 q-1\}$ defined by $f^{*}(u v)=f(u)+f(v)$ is a bijection. In this paper we prove that super subdivision of any cycle $C_{m}$ with $m \geq 3$,ladder, cycle $C_{n}$ for $n \equiv 0(\bmod 4)$ with $K_{1, m}$ and uniform fire cracker are odd harmonious graphs.
\end{abstract}

Keywords : harmonious labeling, odd harmonious labeling, super subdivision of graphs.

AMS Subject Classification (2010) : 05C78 


\section{Introduction}

Throughout this paper by a graph we mean a finite, simple and undirected one. For standard terminology and notation we follow Harary [3]. A graph $G=(V, E)$ with $p$ vertices and $q$ edges is called a $(p, q)-$ graph. The graph labeling is an assignment of integers to the set of vertices or edges or both, subject to certain conditions. An extensive survey of various graph labeling problems is available in [1]. Labeled graphs serves as useful mathematical models for many applications such as coding theory, including the design of good radar type codes, synch-set codes, missile guidance codes and convolution codes with optimal autocorrelation properties. They facilitate the optimal nonstandard encoding of integers. Graham and Sloane [2] introduced harmonious labeling during their study of modular versions of additive bases problems stemming from error correcting codes. A graph $G$ is said to be harmonious if there exists an injection $f: V(G) \rightarrow Z_{q}$ such that the induced function $f^{*}: E(G) \rightarrow Z_{q}$ defined by $f^{*}(u v)=(f(u)+f(v)) \quad(\bmod q)$ is a bijection and $f$ is called harmonious labeling of $G$. The concept of an odd harmonious labeling was due to Liang and Bai [4]. A graph $G$ is said to be odd harmonious if there exists an injection $f: V(G) \rightarrow\{0,1,2, \cdots, 2 q-1\}$ such that the induced function $f^{*}: E(G) \rightarrow\{1,3, \cdots, 2 q-1\}$ defined by $f^{*}(u v)=f(u)+f(v)$ is a bijection. If $f(V(G))=\{0,1,2, \cdots, q\}$ then $f$ is called as strongly odd harmonious labeling and $G$ is called as strongly odd harmonious graph.The odd harmoniousness of graph is useful for the solution of undetermined equations. Several results have been published on odd harmonious labeling and the reader can refer to [5] to [11].

We use the following definitions in the subsequent section.

Definition 1. Let $G$ be a graph with $p$ vertices and $q$ edges. The subdivision graph of $G$ denoted by $S(G)$ is obtained by subdividing every edge of $G$ with a vertex exactly once.

Definition 2. The super subdivision of $G$ denoted by $S S(G)$ is obtained from $G$ by replacing every edge of $G$ by a complete bipartite graph $K_{2, m}$ (where $m$ is an integer).

Definition 3. The arbitrary super subdivision of a graph $G$ denoted by $A S S(G)$ is obtained by replacing each edge of $G$ by a complete bipartite 
graph $K_{2, m_{i}}$ (where $m_{i}$ is any positive integer) in such a way that the end vertices of each $e_{i}$ are merged with two vertices of 2-vertices part of $K_{2, m_{i}}$.

Definition 4. The ladder graph $L_{n}=P_{n} \times P_{2}$ is obtained from the cartesian product of paths $P_{n}$ and $P_{2}$. $L_{n}$ has $2 n$ vertices and $3 n-2$ edges.

Definition 5. The $(m, n)$ - firecracker is denoted by $F_{m, n}$ obtained by the concatenation of $m, n$-stars by linking one leaf from each star.

\section{Main Results}

In this section we prove that super subdivision of any cycle $C_{m}$ with $m \geq 3$, ladder, cycle $C_{n}$ for $n \equiv 0(\bmod 4)$ with $K_{1, m}$ and uniform fire cracker are odd harmonious graphs.

Theorem 2.1. The $(m, n)$ - firecracker graph $F_{m, n}$ is an odd harmonious graph.

Proof. The $(m, n)$ - firecracker graph $F_{m, n}$ has $m(n+1)$ vertices and $m(n+1)-1$ edges.

Let the vertex set be

$V\left(F_{(m, n)}\right)$

$=\left\{v_{1}, v_{2}, \cdots, v_{m}\right\} \cup\left\{v_{1}^{1}, v_{1}^{2}, \cdots, v_{1}^{n}\right\} \cup\left\{v_{2}^{1}, v_{2}^{2}, \cdots, v_{2}^{n}\right\} \cup \ldots \ldots \cup\left\{v_{m}^{1}, v_{m}^{2}, \cdots, v_{m}^{n}\right\}$

and the edge set $E\left(F_{m, n}\right)=\left\{v_{i} v_{i}^{j}: 1 \leq j \leq n, 1 \leq i \leq m\right\} \cup$

$\left\{v_{i}^{n} v_{i+1}^{1}: 1 \leq i \leq m\right.$ and if $i$ is odd $\} \cup\left\{v_{i}^{1} v_{i+1}^{n}: 1 \leq i \leq m\right.$ and if $i$ is even $\}$.

We define a labeling $f: V\left(F_{m, n}\right) \rightarrow\{0,1,2, \cdots, 2(m(n+1)-1)-1\}$ as follows:

$f\left(v_{i}\right)= \begin{cases}(i-1) n+(i-1) & \text { if } i \text { is odd } \\ i n+i-1 & \text { if } i \text { is even; }\end{cases}$

For $1 \leq i \leq m, f\left(v_{i}^{j}\right)=\left\{\begin{array}{ll}(i-1) n+2 j+(i-2), 1 \leq j \leq n & \text { if } i \text { is odd } \\ (i-2) n+2 j+(i-2), 1 \leq j \leq n & \text { if } i \text { is even. }\end{array}\right.$.

The induced edge labels are

$f^{*}\left(v_{i} v_{i}^{j}\right)=(2 i-2) n+2 j+(2 i-3), 1 \leq j \leq n$ and $1 \leq i \leq m ;$

$f^{*}\left(v_{i}^{n} v_{i+1}^{1}\right)=2 i n+2 i-1,1 \leq i \leq m$ and if $i$ is odd; 
$f^{*}\left(v_{i}^{1} v_{i+1}^{n}\right)=2 i n+2 i-1,1 \leq i \leq m$ and if $i$ is even.

In the view of above defined labeling pattern, the resultant graph $F_{(m, n)}$ is odd harmonious.

An odd harmonious labeling of $F_{(4,5)}$ is shown in Figure 1.

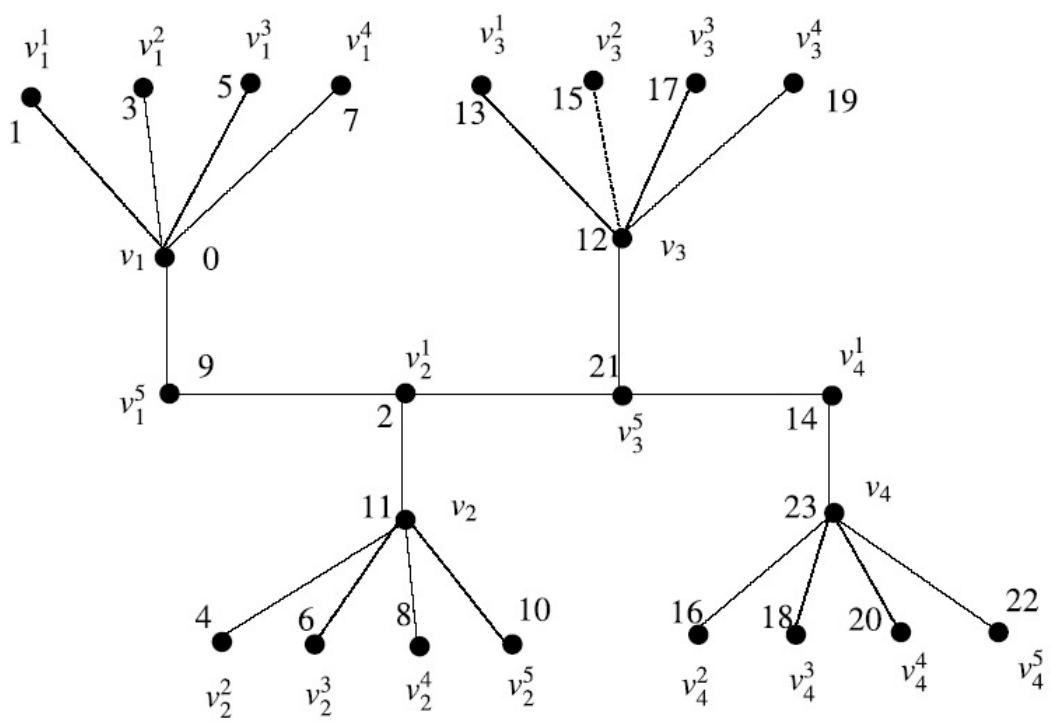

Figure 1:An odd harmonious labeling of $F_{4,5}$.

Theorem 2.2. Let $n$ be an integer with $n \equiv 0(\bmod 4)$ and $m$ is any integer. The graph $G$ with the vertex set $V(G)=\left\{v_{i}, v_{i}^{j}: 1 \leq i \leq n, 1 \leq j \leq m\right\}$ and the edge set $E(G)=\left\{v_{i} v_{i}^{j}: 1 \leq i \leq n, 1 \leq j \leq m\right\} \cup\left\{v_{i}^{m} v_{i+1}^{1}\right.$ and if $i$ is odd, $v_{i}^{1} v_{i+1}^{m}$ and if $i$ is even, $v_{n}^{1} v_{1}^{m}$ is an odd harmonious graph.

Proof. The graph $G$ has $n(m+1)$ vertices and $n(m+1)$ edges.

Define a vertex labeling $f: V(G) \rightarrow\{0,1,2, \cdots, 2 n(m+1)-1\}$ as follows:

For $1 \leq i \leq n$

$f\left(v_{i}\right)=\left\{\begin{array}{ll}(i-1) m+(i-1) & \text { if } 1 \leq i \leq \frac{n}{2}-1 \\ (i-1) m+(i+1) & \text { if } \frac{n}{2}+1 \leq i \leq(n-1)\end{array} \quad\right.$ if $i$ is odd; 
$f\left(v_{i}\right)=i m+(i-1)$ if $i$ is even;

For $1 \leq j \leq m$

$f\left(v_{i}^{j}\right)=\left\{\begin{array}{ll}(i-2) m+2 j+(i-2) & \text { if } i \text { is even } \\ (i-1) m+2 j+(i-2) & \text { if } i \text { is odd }\end{array} \quad 1 \leq i \leq \frac{n}{2} ;\right.$

$f\left(v_{i}^{j}\right)= \begin{cases}(i-2) m+2 j+i & \text { if } i \text { is even } \quad \frac{n}{2}+1 \leq i \leq n \\ (i-1) m+2 j+(i-2) & \text { if } i \text { is odd }\end{cases}$

The induced edge labels are

$f^{*}\left(v_{i} v_{i}^{j}\right)=2(i-1) m+2 j+(2 i-3), 1 \leq j \leq m$ and $1 \leq i \leq \frac{n}{2} ;$

$f^{*}\left(v_{i} v_{i}^{j}\right)=2(i-1) m+2 j+(2 i-1), 1 \leq j \leq m$ and $\frac{n}{2}+1 \leq i \leq n$;

$f^{*}\left(v_{i}^{m} v_{i+1}^{1}\right)=\left\{\begin{array}{ll}2 i m+2 i-1,1 \leq i \leq \frac{n}{2}-1 \\ 2 i m+2 i+1, \frac{n}{2}+1 \leq i \leq(n-1)\end{array} \quad\right.$ if $i$ is odd

$f^{*}\left(v_{i}^{1} v_{i+1}^{m}\right)=\left\{\begin{array}{l}2 i m+2 i-1,2 \leq i \leq \frac{n}{2} \\ 2 i m+2 i+1, \frac{n}{2}+2 \leq i \leq(n-2)\end{array} \quad\right.$ if $i$ is even;

Also $f^{*}\left(v_{n}^{1} v_{1}^{m}\right)=n m+n+1$.

In the view of above defined labeling pattern the graph $G$ admits an odd harmonious labeling. 
An odd harmonious labeling of a cycle $C_{4}$ with $K_{1,3}$ is shown in Figure 2.

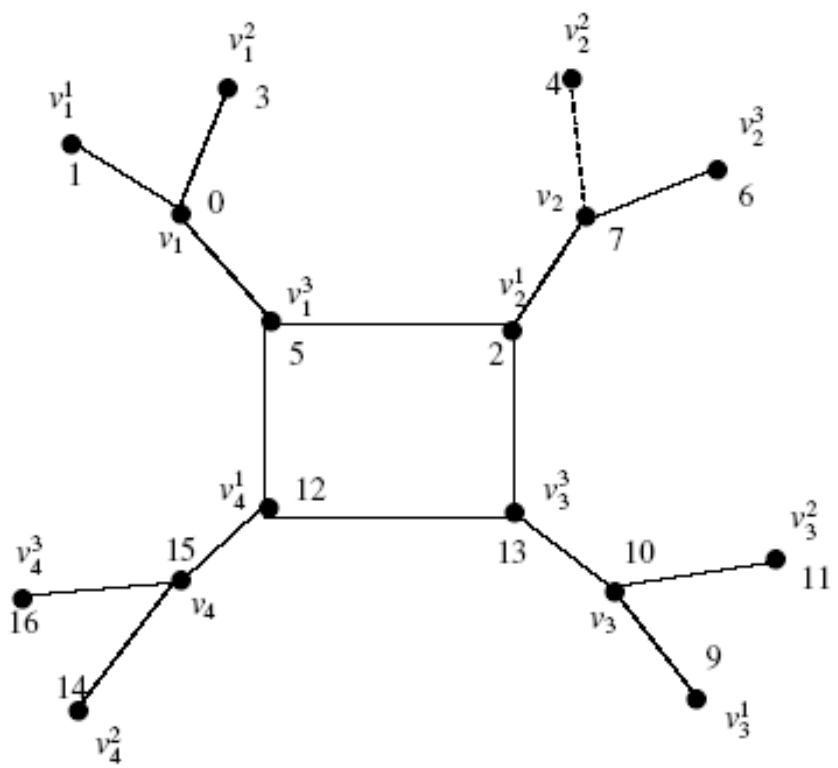

Figure2: An odd harmonious labeling of $C_{4}$ with $K_{1,3}$.

Theorem 2.3. The arbitrary super subdivision of a cycle $C_{m}, m \geq 3$ is an odd harmonious graph.

Proof. Let the edges of $C_{m}$ be $e_{1}, e_{2}, \ldots . e_{m}$. Let $G$ be the graph obtained by replacing the edges $e_{i}, 1 \leq i \leq(m-1)$ by a graphs $K_{2, n_{i}}$ and the edge $e_{m}$ by $K_{2, m-1}$, where $n_{1}, n_{2}, \ldots, n_{m-1}$ are integers.

Let the vertex set of $G$ be $V=\left\{v_{1}, v_{2}, \ldots, v_{m}\right\} \cup\left\{u_{1}, u_{2}, \ldots, u_{n_{1}}\right\} \cup\left\{u_{n_{1}+1}, u_{n_{1}+2}, \ldots, u_{n_{1}+n_{2}}\right\} \cup \ldots$ $\cup\left\{u_{n_{1}+n_{2}+\ldots .+n_{m-1}+1}, \ldots ., u_{n_{1}+n_{2}+\ldots . .+n_{m-1}+n_{m}}\right\}$.

Then the graph $G$ has $m+n_{1}+n_{2}+\ldots+n_{m-1}+(m-1)$ vertices and $2\left(n_{1}+n_{2}+\ldots .+n_{m-1}\right)+2(m-1)$ edges.

We define a labeling $f: V(G) \rightarrow\left\{0,1,2, \ldots 4\left(n_{1}+n_{2}+\ldots .+n_{m-1}+(m-1)\right)-1\right\}$ as follows:

$$
f\left(v_{i}\right)=2(i-1), \text { if } 1 \leq i \leq m ;
$$

$f\left(u_{i}\right)=4 i-3$, if $1 \leq i \leq n_{1}$; 
$f\left(u_{i}\right)=4 i-[2(k+1)+1]$, if $n_{1}+n_{2}+\ldots+n_{k}+1 \leq i \leq n_{1}+n_{2}+\ldots+n_{k+1}$ and $1 \leq k \leq(m-2)$;

$f\left(u_{i}\right)=4\left(n_{1}+n_{2}+\ldots+n_{m-1}\right)+1$ if $i=n_{1}+n_{2}+\ldots .+n_{m-1}+1$ and $f\left(u_{i}\right)=f\left(u_{i-1}\right)+2$ if $n_{1}+n_{2}+\ldots+n_{m-1}+2 \leq i \leq n_{1}+n_{2}+\ldots .+n_{m}$.

The induced edge labels are

$f^{*}\left(v_{1} u_{i}\right)=4 i-3,1 \leq i \leq n_{1}$

$f^{*}\left(v_{k+1} u_{i}\right)=4 i-3, n_{1}+n_{2}+\ldots+n_{k}+1 \leq i \leq n_{1}+n_{2}+\ldots+n_{k+1}$ and $1 \leq k \leq(m-2)$;

Also $f^{*}\left(u_{i} v_{2}\right)=4 i-1,1 \leq i \leq n_{1}$;

$f^{*}\left(u_{i} v_{k+1}\right)=4 i-1, n_{1}+n_{2}+\ldots+n_{k-1}+1 \leq i \leq n_{1}+n_{2}+\ldots+n_{k}$ and $2 \leq k \leq(m-1)$

$f^{*}\left(v_{m} u_{n_{1}+n_{2}+\ldots+n_{m-1}+k}\right)=2(m-1)+4\left(n_{1}+n_{2}+\ldots+n_{m-1}\right)+[2(k)-1]$ and $1 \leq k \leq(m-1)$;

$f^{*}\left(u_{n_{1}+n_{2}+\ldots+n_{m-1}+k} v_{1}\right)=4\left(n_{1}+n_{2}+\ldots+n_{m-1}\right)+[2(k)-1]$ and $1 \leq k \leq$ $(m-1)$.

In the view of above defined labeling pattern, the super subdivision of cycle $C_{m}, m \geq 3$ admits an odd harmonious labeling. 
An odd harmonious labeling of super subdivision of a cycle $C_{3}$ is shown in Figure 3.

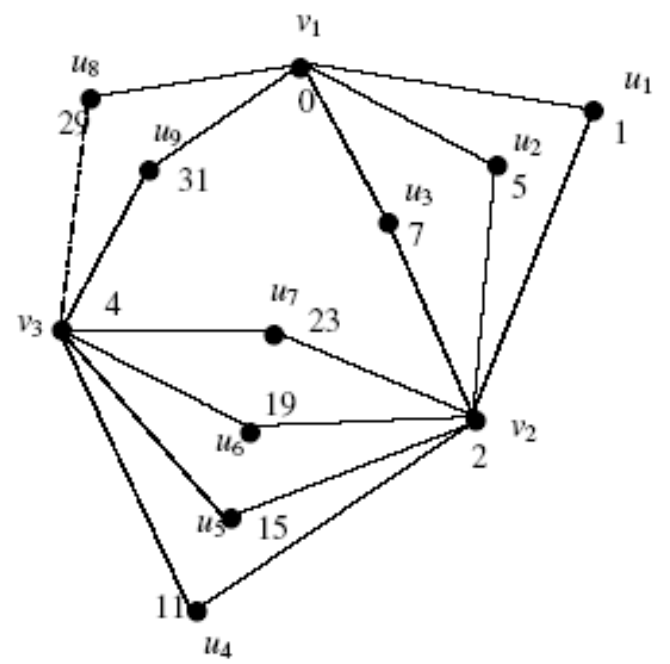

Figure 3:An odd harmonious labeling of super subdivision of cycle $C_{3}$.

Theorem 2.4. The super subdivision of ladder graph is odd harmonious.

Proof. This graph $G$ contains $(3 n-2) m+2 n$ vertices and $(3 n-2) 2 m$ edges.

Let the vertex set be

$V=\left\{v_{1}, v_{2}, \ldots, v_{2 n}\right\} \cup$

$\left\{c_{1}, c_{2}, \ldots, c_{m}, c_{m+1}, \ldots, c_{2 m}, c_{2 m+1}, \ldots, c_{3 m}, c_{3 m+1}, \ldots, c_{4 m}, \ldots, c_{(3 n-2) m}\right\}$. lows:

We define a labeling $f: V(G) \rightarrow\{0,1,2, \ldots .2((3 n-2) 2 m)-1\}$ as fol-

$f\left(v_{j}\right)=2 m(j-1)$, if $1 \leq j \leq 2 n$;

$f\left(c_{i}\right)=(2 i-1), 1 \leq i \leq 2 m$;

$f\left(c_{i}\right)=(2 i-1)+(2 k-2) m$ if $(3 k-4) m+1 \leq i \leq(3 k-1) m$ and $k=2,3, \ldots,(n-1)$;

$f\left(c_{i}\right)=(2 i-1)+(2 k-2) m$ if $k=n$ and $(3 k-4) m+1 \leq i \leq(3 k-2) m$.

The induced edge labels are 
$f^{*}\left(v_{j} c_{i}\right)=2 m(j-1)+2 i-1,1 \leq i \leq 2 m$, here $v_{j}$ 's are adjacent with $C_{i}$ 's where $1 \leq i \leq 2 m$;

$f^{*}\left(v_{j} c_{i}\right)=2 m(j-1)+2 i-1+(2 k-2) m,(3 k-4) m+1 \leq i \leq(3 k-1) m$, $k=2,3, \ldots,(n-1)$,

here $v_{j}$ 's are adjacent with $C_{i}$ 's where $(3 k-4) m+1 \leq i \leq(3 k-1) m$;

When $k=n, f^{*}\left(v_{j} c_{i}\right)=2 m(j-1)+2 i-1+(2 k-2) m,(3 k-4) m+1 \leq$ $i \leq(3 k-2) m$,

here $v_{j}$ 's are adjacent with $C_{i}$ 's where $(3 k-4) m+1 \leq i \leq(3 k-2) m$.

In the view of defined labeling pattern, the super subdivision of ladder graph admits an odd harmonious labeling.

An odd harmonious labeling of the super subdivision of ladder with $n=2$ and $m=3$ is shown in Figure 4 .

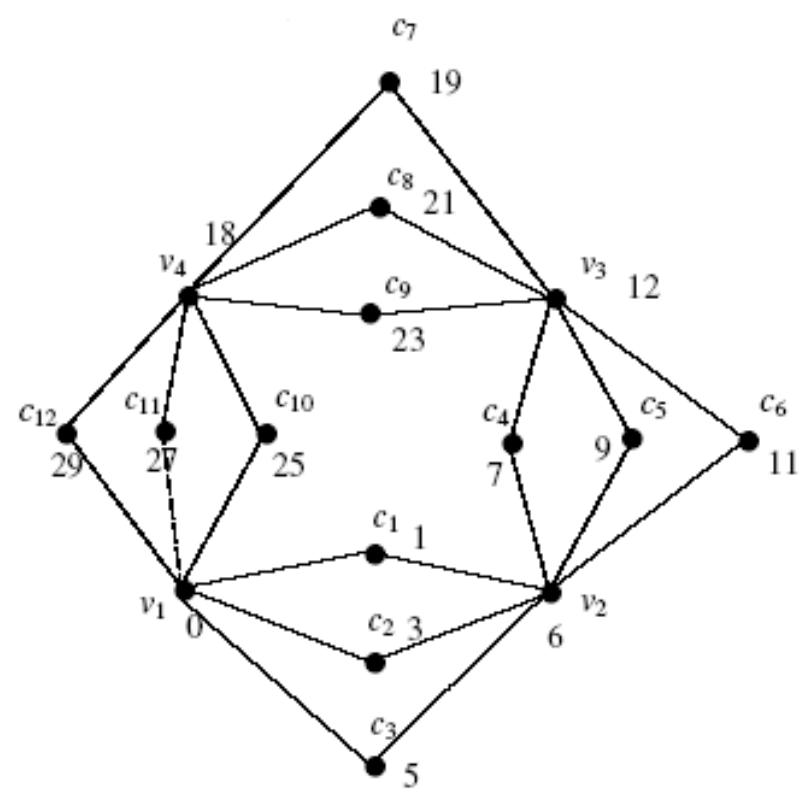

Figure 4:An odd harmonious labeling of super subdivision of ladder with $n=2$ and $m=3$ 


\section{References}

[1] J. A. Gallian, A Dynamic Survey of Graph Labeling, The Electronics Journal of Combinatorics, (2017), \# DS6.

[2] R. L. Graham and N. J. A. Sloane, On Additive bases and Harmonious Graphs, SIAM J. Algebr. Disc. Meth., 4, pp. 382-404, (1980).

[3] F. Harary, Graph Theory, Addison-Wesley, Massachusetts, (1972).

[4] Z. Liang, Z. Bai, On the Odd Harmonious Graphs with Applications, J. Appl. Math. Comput., 29, pp. 105-116, (2009).

[5] P. Jeyanthi, S. Philo and Kiki A. Sugeng, Odd harmonious labeling of some new families of graphs, SUT Journal of Mathematics, Vol. 51, No. 2, pp. 53-65, (2015).

[6] P. Jeyanthi, S. Philo, Odd Harmonious Labeling of Some Cycle Related Graphs, Proyecciones Journal of Mathematics, Vol. 35, No. 1, pp. 8598, (2016).

[7] P. Jeyanthi, S. Philo, Odd Harmonious Labeling of Plus Graphs, Bulletin of the International Mathematical Virtual Institute, Vol. 7, pp. 515-526, (2017).

[8] P. Jeyanthi, S. Philo, Maged Z. Youssef, Odd Harmonious Labeling of Grid Graphs, Proyecciones Journal of Mathematics, to appear.

[9] P. Selvaraju, P. Balaganesan and J. Renuka, Odd Harmonious Labeling of Some Path Related Graphs, International J. of Math.Sci. and Engg. Appls., 7 (III), pp. 163-170, (2013).

[10] S. K. Vaidya and N. H. Shah, Some New Odd Harmonious Graphs, International Journal of Mathematics and Soft Computing, 1, pp. 9-16, (2011).

[11] S. K. Vaidya, N. H. Shah, Odd Harmonious Labeling of Some Graphs, International J.Math. Combin., 3, pp. 105-112, (2012). 


\section{P. Jeyanthi}

Research Centre,

Department of Mathematics,

Govindammal Aditanar College for Women,

Tiruchendur - 628 215, Tamil Nadu,

India

e-mail: jeyajeyanthi@rediffmail.com

\section{S. Philo}

Research Scholar,

Reg. No: 12193,

Manonmaniam Sundaranar University

Abishekappatti, Tirunelveli - 627012

India

e-mail: lavernejudia@gmail.com

and

\section{K. Siddiqui}

Department of Mathematics,

COMSATS University Islamabad,

Sahiwal Campus,

Pakistan

e-mail: kamransiddiqui75@gmail.com 